\title{
Computational screening of ionic liquids as solvents for reprocessing of spent nuclear fuel
}

\author{
Yusuf A. Z. ${ }^{1 *}$, Zakir A. ${ }^{2}$, Mustapha S..$^{3}$, Halima S. A. ${ }^{3}$ and Nuhu M. ${ }^{3}$ \\ ${ }^{1}$ Nigerian National Petroleum Cooperation (NNPC), Nigeria. \\ ${ }^{2}$ Human Physiology Department, Ahmadu Bello University Zaria. \\ ${ }^{3}$ Chemical Engineering Department, Ahmadu Bello University Zaria.
}

Accepted 7 January, 2014

COSMO-RS method was used in screening 2106 ionic liquids as alternate solvents to volatile $\mathrm{n}$ dodecane used in PUREX. The distribution coefficients of uranylnitrate in ionic liquids/aqueous nitric acid biphasic system and the solubility of the ionic liquids in water were determined using COSMO-RS. Seventeen ionic liquids based on tetrafluoroborate $\mathrm{BF}_{4}$, were the most hydrophobic than others screened with hexamethylguanidiumtetrafluoroborate having the highest distribution coefficient. Their distribution coefficients were higher than that of the convention $\mathrm{n}$-dodecane used for separation of uranium and plutonium from spent nuclear fuel but having more hydrophilicity than $\mathrm{n}$-dodecane. This shows that ionic liquids can be used as alternate solvents in PUREX.

Key words: Ionic liquids, spent nuclear fuel (SNF), conductor-like screening model (COSMO-RS), Plutonium uranium extraction (PUREX), COSMOtherm, n-dodecane, n-tributylphosphate (TBP).

\section{INTRODUCTION}

The products contained in SNF are radioactive with halflives from days to millions of years which give a lot of threat to human health and environment (Terry, 2008). Uranium and Plutonium are conventionally recovered through PUREX process. PUREX, an abbreviation for Plutonium (P) Uranium (UR) Extraction (EX), makes use of about $30 \%$ tri- $n$-butyl phosphate (TBP) with Kerosene or pure $n$-Dodecaneas diluents (Sung et al., 2010). In this process, Uranium and Plutonium are extracted as $\mathrm{UO}_{2}{ }^{2+}$ and $\mathrm{Pu}^{4+}$ and then converted to $\mathrm{UO}_{2}$ and $\mathrm{PuO}_{2}$. Aside from uranium and plutonium, various fission products can be recovered from SNF. Thus some extraction processes have been developed using different extracting agents to recover fission products from PUREX raffinate. The extraction processes developed for SNF treatment and recovery, utilize organic solvents that are volatile, hazardous, toxic, flammable, and unstable towards the radiation and heat generated by the SNF (Sung et al., 2010). Since the undesirable properties of these conventional solvents may pose several threats and problems both to plant operation and to environment, several researches are ongoing in search of alternate safer solvents such as ionic liquids (ILs) which have unique physical and chemical properties, such as specific solvent abilities, negligible vapor pressures, non flammability, thermal and radiological stability and broad liquid temperature ranges among many. They have led to promising applications as environmentally benign solvents that can be possible candidates to replace conventional organic solvents (Sung et al., 2010).

During the past 10 years, ionic liquids (ILs) have received increased attention. They are organic salts, and 
their chemical and physical properties can be tailored by the selection of an anion and cation (Wasserscheid, 2000). Therefore, it is possible to generate a huge number of different ionic liquids, each with specific properties. Despite the interest, accurate thermodynamic data of ionic liquids and their mixtures are still rare and to exploit the potential of these new substances, it would be of great value to have prediction methods that can reliably predict the thermodynamic properties of ionic liquids and their mixtures. This would help to scan the growing set of already known ILs in order to find suitable candidates for a certain task or to design new ILs for special applications (Geetanjali and Kumar, 2008). Group contribution methods, which are the most widely used theoretical models are not applicable because group interactions parameters are not available at present for ionic liquids and the group contribution concept is not suitable to handle the long-range interactions in ionic compounds (Hamad and Sumon, 2008). Monte Carlo simulations and molecular dynamics need appropriate force-fields for the treatment of ionic liquids, which have to be developed (Hamad and Sumon, 2008). This work will present the application of a continuum solvation based thermodynamic prediction model, Conductor-like Screening Model for Real Solvents, COSMO-RS for prediction of thermo-physical data which is a computational approach independent of experimental data and with general applicability (Eckert and Klamt, 2002).

The aim of the research is to computationally screen suitable hydrophobic ionic liquids as solvents for the extraction of Uranium from spent nuclear fuel using COSMO -RS method by determining the distribution coefficients of uranyl nitrate in hydrophobic ionic liquids/ aqueous nitric acid biphasic solution and determination the solubilityof the ionic liquids in water. The work will be limited to the application of COSMO-RS, a thermodynamic model which uses statistical thermodynamic approach in determination of thermodynamic properties based on results obtained from quantum chemical/COSMO computation for the screening of ionic liquids for Uranium Extraction from spent nuclear fuel.

\section{MATERIALS AND METHODS}

The following are the materials used in carrying out the research work:
a) System Softwares
i) Linux operating system
ii) Windows 7 operating system
b) Application Softwares
i) Chem draw 3D ultra 8.0/ Marvin sketch software.
ii) TURBOMOLE 5.6 Program Package (LINUX VERSION).
iii) COSMOthermSoftware.

The following are the methods used in carrying out the research work:

1) The Marvin sketch software is first used in sketching the structure of each individual molecule. The environment has a drawing kit including all elements in the periodic table and heterocyclic structures for easy sketch of the molecular structures. Marvin sketch software operates on windows. The file generated is saved in xyz format and is used as an input file for TURBOMOLE to perform the quantum chemical/COSMO calculation.

2) The TURBOMOLE uses the xyz file generated from Marvin sketch to perform quantum chemical/COSMO calculation to generate the screening charge densities of the molecules. The screening charge is a microscopic property of a molecule like internal energy and it is the main parameter used in the COSMORS model. The quantum chemical/COSMO computation on TURBOMOLE 5.6 is parameterized using DFT-level of computation utilizing BP-functional on TZVP basis set. The file is saved as a COSMO file and an input file to COSMOtherm to perform the statistical thermodynamics and to determine the screening charge density profiles and chemical potentials of the individual molecules (Klamt, 1995). The higher the specification of the computer machine, the faster the computation of the screening charges of the molecules.

3) A database is created for all the COSMO files generated using the TURBOMOLE program software.

4) The screening charge density profiles and the chemical potentials of the molecules are determined using COSMOtherm. The COSMOtherm is the software that solves the COSMO-RS model based on self consistency field algorithm (SCF) to statistically determine the screening charge density profiles and the chemical potentials of the molecules (Klamt, 1995).

5) The 81 cations and 26 anions were combined to generate 2106 ionic liquids (Table 1).

6) The solubility of the ionic liquids in water is determined from cosMOtherm using the equation;

$\log _{10}\left(x_{j}^{\text {sol }}\right)=\left[\mu_{j}^{(p)}-\mu_{j}^{(t)}-\max \left(0, \Delta G_{\text {fus }}\right)\right] /(R T \ln (10))$

Where $\Delta \mathrm{G}_{\text {fus }}$ is the heat of fusion per mole. If the compound is solid, the energy change of a compound from the super cooled liquid state to the ordered solid state has to be taken into account, that is, the solutes Gibbs free energy of fusion $\Delta G_{\text {fus }}$ (or equivalently its Gibbs free energy of crystallization $\Delta \mathrm{G}_{\text {cryst }}=-\Delta \mathrm{G}_{\text {fus }}$ ) has to be either given and computed from experimental data or estimated by cosMOtherm. For a liquid, it is automatically specified but for solids it has to be determined using QSPR and then specified manually.

$\mathrm{T}=$ Temperature in degree Celsius

$\mathrm{R}=$ molar gas constant in $\mathrm{KJ} / \mathrm{mol} /$ Kelvin

$\mu_{\mathrm{j}}^{(i)}=$ chemical potential of solute $\mathrm{j}$ in solvent $\mathrm{i}$ in energy $/ \mathrm{mole}$

$\mu_{j}^{(P)}=$ chemical potential of pure compound $\mathrm{j}$ in energy $/ \mathrm{mole}$

$x_{j}=$ mole fraction of the solubility of solutes in water (dimensionless).

7) The distribution coefficient and octanol-water partition coefficients are determined from COSMOtherm using the equation:

$\left.\left.\log _{10}(p)=\log _{10}\left[\exp \left(\mu_{j}^{(1)}-\mu_{j}^{(t)}\right)\right] / R T\right) . V_{1} / V_{2}\right)$

Where $v_{1}$ and $v_{2}$ are volume quotients of phase 1 and phase 2

$\mu_{\mathrm{j}}^{(1)}=$ chemical potential of uranyl nitrate in phase 1 in energy/mole

$\mu_{j}{ }^{(2)}=$ chemical potentials of uranyl nitrate in phase 2 in energy/mole

$\mathrm{P}=$ partition coefficient (dimensionless). 
Table 1. List of cations and anions.

\begin{tabular}{|c|c|c|c|}
\hline $\mathbf{S} / \mathbf{N}$ & \multicolumn{3}{|c|}{ Cations } \\
\hline 1 & 1,1-dimethyl-pyrrolidinium & 42 & methyl-trioctyl-ammonium \\
\hline 2 & 1,1-dipropyl-pyrrolidinium & 43 & tetra-methyl ammonium \\
\hline 3 & 1-ethyl-1-methyl-pyrrolidinium & 44 & tetra-ethyl ammonium \\
\hline 4 & 1-butyl-1-methyl-pyrrolidinium & 45 & tetra-n-butyl ammonium \\
\hline 5 & 1-butyl-1-ethyl-pyrrolidinium & 46 & benzyl-triphenyl-phosphonium \\
\hline 6 & 1-hexyl-1-methyl-pyrrolidinium & 47 & tetrabutyl-phosphonium \\
\hline 7 & 1-octyl-1-methyl-pyrrolidinium & 48 & trihexyl-tetradecyl-phosphonium \\
\hline 8 & 3-methyl-imidazolium & 49 & triisobutyl-methyl-phosphonium \\
\hline 9 & 1-butyl-imidazolium & 50 & guanidinium \\
\hline 10 & 1,3-dimethyl-imidazolium & 51 & hexamethylguanidinium \\
\hline 11 & 1-ethyl-3-methyl-imidazolium & 52 & N,N,N,N,N-pentamethyl-N-isopropyl-guanidinium \\
\hline 12 & 1-butyl-3-methyl-imidazolium & 53 & N,N,N,N,N-pentamethyl-N-propyl-guanidinium \\
\hline 13 & 1-pentyl-3-methyl-imidazolium & 54 & N,N,N,N-tetramethyl-N-ethylguanidinium \\
\hline 14 & 1-hexyl-3-methyl-imidazolium & 55 & S-ethyl-N,N,N,N-tetramethylisothiouronium \\
\hline 15 & 1-octyl-3-methyl-imidazolium & 56 & O-ethyl-N,N,N,N-tetramethylisouronium \\
\hline 16 & 1-decyl-3-methyl-imidazolium & 57 & O-methyl-N,N,N,N-tetramethylisouronium \\
\hline 17 & 1-dodecyl-3-methyl-imidazolium & 58 & N-butyl-isoquinolinium \\
\hline 18 & 1-tetradecyl-3-methyl-imidazolium & 59 & morpholinium \\
\hline 19 & 1-hexadecyl-3-methyl-imidazolium & 60 & methylmorpholinium \\
\hline 20 & 1-octadecyl-3-methyl-imidazolium & 61 & dimethylmorpholinium \\
\hline 21 & 1-benzyl-3-methyl-imidazolium & 62 & $\mathrm{~N}$-fluoropropoxy-methylmorpholinium \\
\hline 22 & 1-ethyl-2-3-methyl-imidazolium & 63 & O-ethyl-tetrapropylisouronium \\
\hline 23 & 1-propyl-2-3-methyl-imidazolium & 64 & O-hydro-tetraethylisouronium \\
\hline 24 & 1-butyl-2-3-methyl-imidazolium & 65 & O-hydro-tetramethylisouronium \\
\hline 25 & 1-hexyl-2-3-methyl-imidazolium & 66 & O-methyl-tetraethylisouronium \\
\hline 26 & 1-hexadecyl-2-3-methyl-imidazolium & 67 & O-methyl-tetrapropylisouronium \\
\hline 27 & 1-methyl-3-(3-phenyl-propyl)-imidazolium & 68 & O-propyl-tetramethylisouronium \\
\hline 28 & 4-methyl-n-butylpyridinium & 69 & O-propyl-tetrapropylisouronium \\
\hline 29 & 1-ethyl-pyridinium & 70 & O-butyl-tetramethylisouronium \\
\hline 30 & 1-butyl-pyridinium & 71 & O-ethyl-tetraethylisouronium \\
\hline 31 & 1-hexyl-pyridinium & 72 & diethyl-dimethylammonium \\
\hline 32 & 1-octyl-pyridinium & 73 & dimethylammonium \\
\hline 33 & 1-butyl-3-ethyl-pyridinium & 74 & ethyl-trimethylammonium \\
\hline 34 & 1-butyl-3-methyl-pyridinium & 75 & methyl-triethylammonium \\
\hline 35 & 1-butyl-4-methyl-pyridinium & 76 & tetra-ethyl ammonium \\
\hline 36 & 1-hexyl-3-methyl-pyridinium & 77 & tetramethylammonium \\
\hline 37 & 1-hexyl-4-methyl-pyridinium & 78 & trimethylammonium \\
\hline 38 & 3-methyl-1-octyl-pyridinium & 79 & n-methyldiazabicyclo-undec-7-enium \\
\hline 39 & 4-methyl-1-octyl-pyridinium & 80 & n-ethyldiazabicyclo-undec-7-enium \\
\hline 40 & 1-butyl-3,4-dimethyl-pyridinium & 81 & n-hexyldiazabicyclo-undec-7-enium \\
\hline 41 & 1-butyl-3,5-dimethyl-pyridinium & & \\
\hline $\mathbf{S} / \mathbf{N}$ & \multicolumn{3}{|c|}{ Anions } \\
\hline 1 & Tetrafluoroborate & 14 & bissalicylatoborate \\
\hline 2 & Hexafluorophosphate (vi) & 15 & tetracyanoborate \\
\hline 3 & methyl sulfate & 16 & bis(2,4,4-trimethylpentyl)phosphinate \\
\hline 4 & chlorate & 17 & bis-pentafluoroethyl-phosphinate \\
\hline 5 & bromate & 18 & bis(trifluoromethylsulfonyl)methane \\
\hline 6 & lodide & 19 & decanoate \\
\hline 7 & formate & 20 & tosylate \\
\hline
\end{tabular}


Table 1. Contd.

\begin{tabular}{c|lrll} 
& toluene-4-sulfonate & bis(pentafluoroethylsulfonyl)imide \\
9 & trifluoromethane-sulfonate & 22 & n-methyl-n-butylcarbamate \\
10 & tris(nonafluorobutyl)trifluorophosphate & 23 & n-methyl-n-propylcarbamate \\
11 & tris(pentafluoroethyl)trifluorophosphate & 24 & Nitrate \\
12 & bisbiphenyldiolatoborate & 25 & Thiocyanate \\
13 & bisoxalatoborate & 26 & Methylphosphonate \\
\hline
\end{tabular}

\section{Post processing}

From the properties determined, criteria for the selection of suitable ionic liquids were imposed. These are based on the following criteria;

\section{Hydrophobicity}

Out of the 2106 ionic liquids, those with mole fraction solubility of 1 are considered hydrophilic while those with mole fraction solubility of zero are considered as hydrophobic ionic liquids.

\section{Comparable partition coefficient with n-dodecane}

Those ionic liquids with distribution coefficient greater than or equal to that of $n$-dodecane were recommended for screening. Similarly, those with solubility mole fraction of less than 1 are considered partially miscible with water while those with solubility mole fraction of 1 are considered fully miscible with water (hydrophilic). Those ionic liquids that satisfy criteria 1 and 2 were recommended. Using these criteria, 111 ionic liquids were screened out of the in the database. 17 ionic liquids based on $\mathrm{BF}_{4}$ are the most hydrophobic. The 17 ionic liquids are given below:

1) 1,1-dimethyl-pyrrolidinium tetrafluoroborate

2) 1-ethyl-1-methyl-pyrrolidinium tetrafluoroborate

3) tetra-ethylammoniumtetrafluoroborate

4) hexamethylguanidiniumtetrafluoroborate

5)

$\mathrm{N}, \mathrm{N}, \mathrm{N}, \mathrm{N}, \mathrm{N}$-pentamethyl-N-isopropyl-

guanidiniumtetrafluoroborate

6) N,N,N,N,N-pentamethyl-N-propyl-guanidiniumtetrafluoroborate

7) S-ethyl-N,N,N,N-tetramethylisothiouroniumtetrafluoroborate

8) O-ethyl-N,N,N,N-tetramethylisouroniumtetrafluoroborate

9) O-methyl-N,N,N,N-tetramethylisouroniumtetrafluoroborate

10) O-methyl-tetraethylisouroniumtetrafluoroborate

11) O-propyl-tetramethylisouroniumtetrafluoroborate

12) O-butyl-tetramethylisouroniumtetrafluoroborate

13) O-ethyl-tetraethylisouroniumtetrafluoroborate

14) methyl-triethylammoniumtetrafluoroborate

15) n-methyldiazabicyclo-undec-7-eniumtetrafluoroborate

16) n-ethyldiazabicyclo-undec-7-eniumtetrafluoroborate

17) tetra-methylammoniumtetrafluoroborate

\section{RESULTS AND DISCUSSION}

\section{Data validation of solubility}

The experimental solubility in water values of some solvents determined was compared with that of COSMORS method. The correlation factor obtained was 0.994 which shows a good correlation between the experimental and that of the model. This is a validation of the model for determination of solubility of compounds in water. Figure 1 gives the comparison between the experimental and the model results. The experimental results were obtained from Laurie (2004) and Felicia et al. (2009).

\section{Evaluation of the distribution coefficient and solubility of $\mathbf{n}$-dodecane and the screened ILs}

The distribution coefficient of uranylnitrate in $\mathrm{n}$-dodecane /aqueous nitric acid biphasic system and that of uranylnitrate in ionic liquids/aqueous nitric acid biphasic system was determined using COSMO-RS method. The solubility of the $\mathrm{n}$-dodecane and ionic liquids in water was also determined to determine the hydrophobicity of the ionic liquids and the n-dodecane. As shown in Figure 2, the solubility of $n$-dodecane in water is 0.0072 and is the most hydrophobic among the solvents and this gives its advantage of easier recycle and recovery. This is because the more hydrophobic the solvent is, the better the separation with the aqueous phase. Among the ionic liquids screened, 17 of them are the most hydrophobic closest to n-dodecane with solubility mole fraction of less than 0.1. Although they have comparable or higher distribution coefficient with the conventional solvent, ndodecane. The advantage of higher distribtution coefficient is the use of less number of stages during continuous extraction. It can also be seen that some ionic liquids give high distribution coefficient but more miscible with water. It has been reported that hydrophilic ionic liquids forms complex with uranyl nitrate via anionic and cationic complexes with contamination of the aqueous phase and this is a limitation in the application of these types of ionic liquids for this application despite the high distribution coefficient (Binnemans, 2007). Hydrophobic ionic liquids on the other hand, forms complex via neutral complexes without contamination of the aqueous phase (Binnemans, 2007).

\section{Effect of nitric acid concentration}

Nitric acid concentration affects the distribution coefficient 


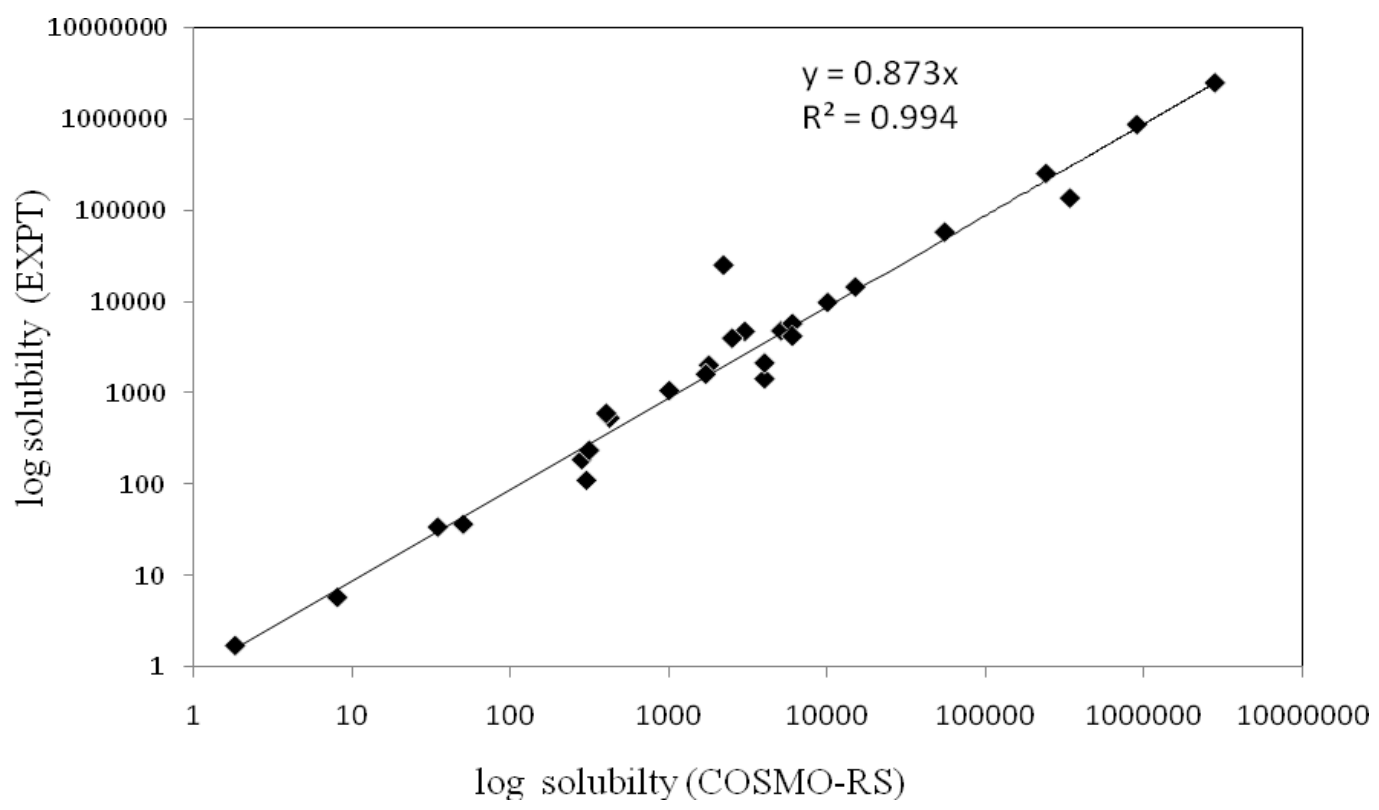

Figure 1. Comparison of experimental solubility values against COSMO-RS.

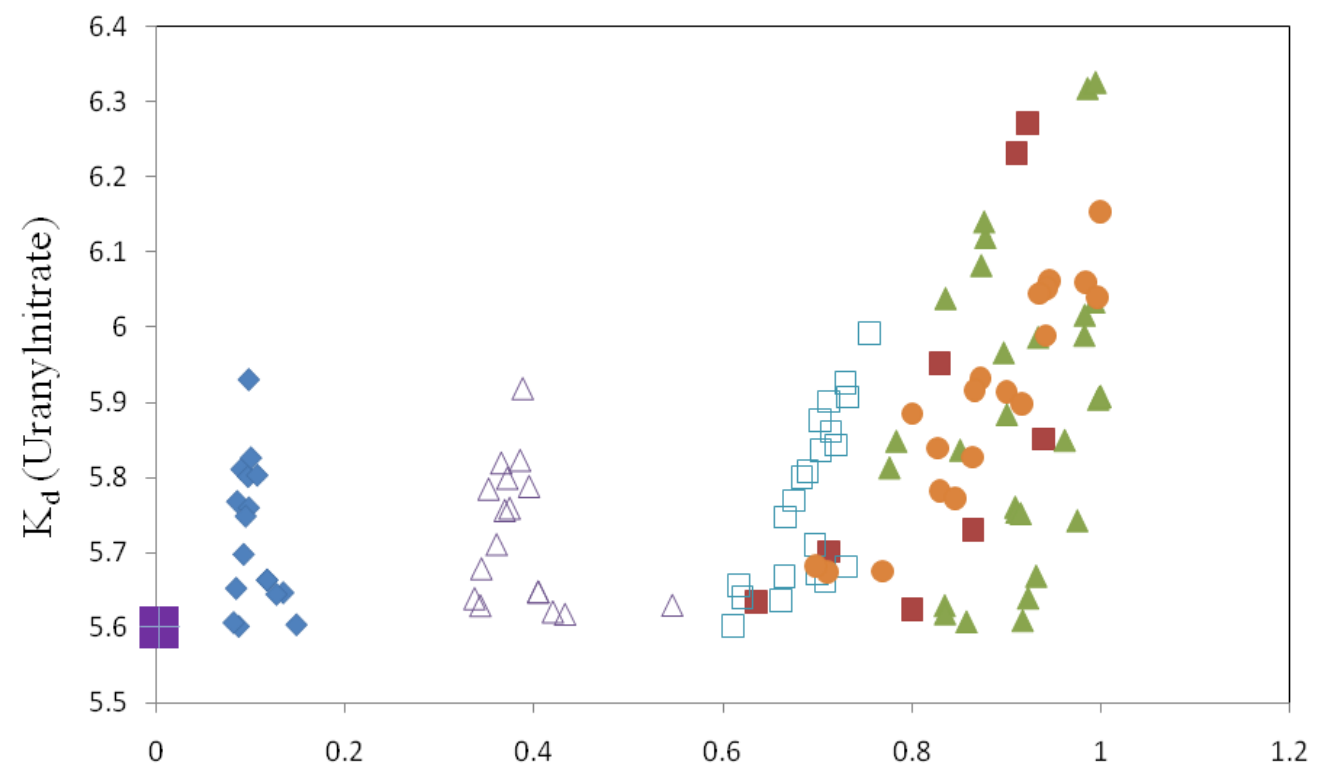

Solubility of solvents in $\mathrm{H}_{2} \mathrm{O}$ (mole fraction)

Figure 2. $K_{d}$ (Uranyl nitrate) against the solubility of solvents in water.

$\checkmark \mathrm{Bf}_{4}$ based ILs $\triangle \mathrm{TF}_{2} \mathrm{~N}$ based ILs Thiocyanate based ILs

- lodo based ILs $\square$ pentafluoroethylphosphinate based ILs

$\triangle$ Methylsulfate based ILs $n$-dodecane (Reference Solvent).

of the uranyl nitrate in the ionic liquid/aqueous nitric acid biphasic system as can be seen in Figure 3 . The distribution coefficient increases with increase in nitric acid concentration from 0.2 to 4 molar and then slightly decreases to 8 molar. This is because with increase in nitric acid concentration, the solubility of the solute in the 


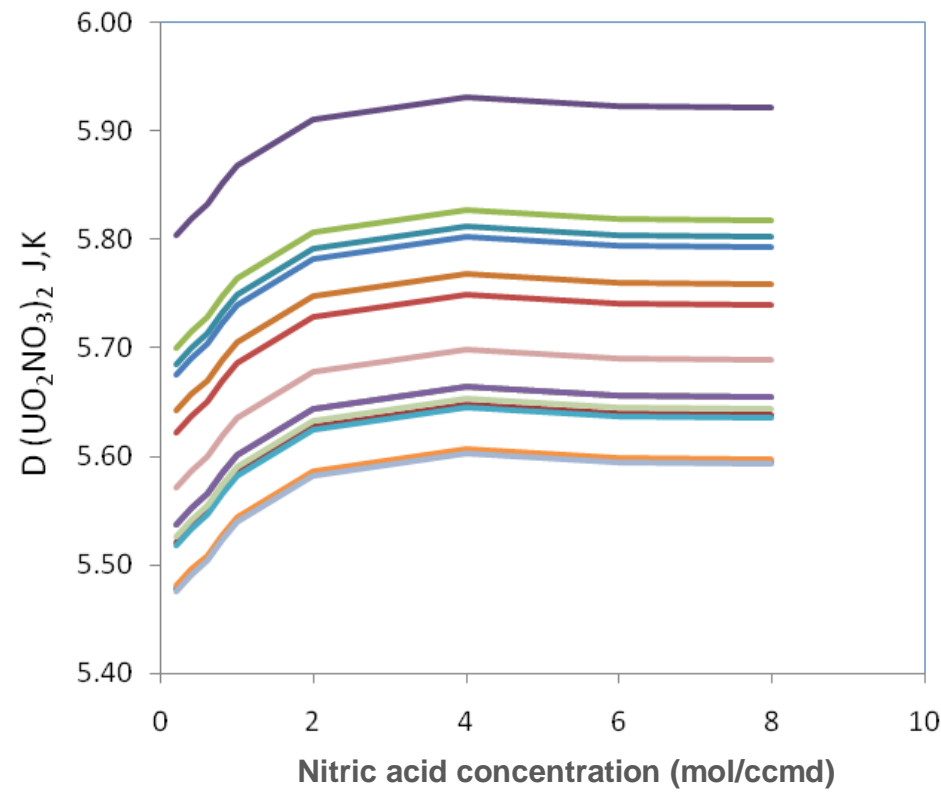

\author{
D= DISTRIBUTION COEFFICIENT \\ $\mathrm{J}=$ AQUEUOS NITRIC ACD \\ $\mathrm{K}=$ IONIC LIQUIDS
}

$\left(\mathrm{UO}_{2} \mathrm{NO}_{3}\right)_{2}=$ URANYL NITRATE

Figure 3. Effect of nitric acid concentration on the $\mathrm{D}\left(\mathrm{UO}_{2} \mathrm{NO}_{3}\right)_{2} \mathrm{~J}, \mathrm{~K}$ of the 17 selected ionic liquids.

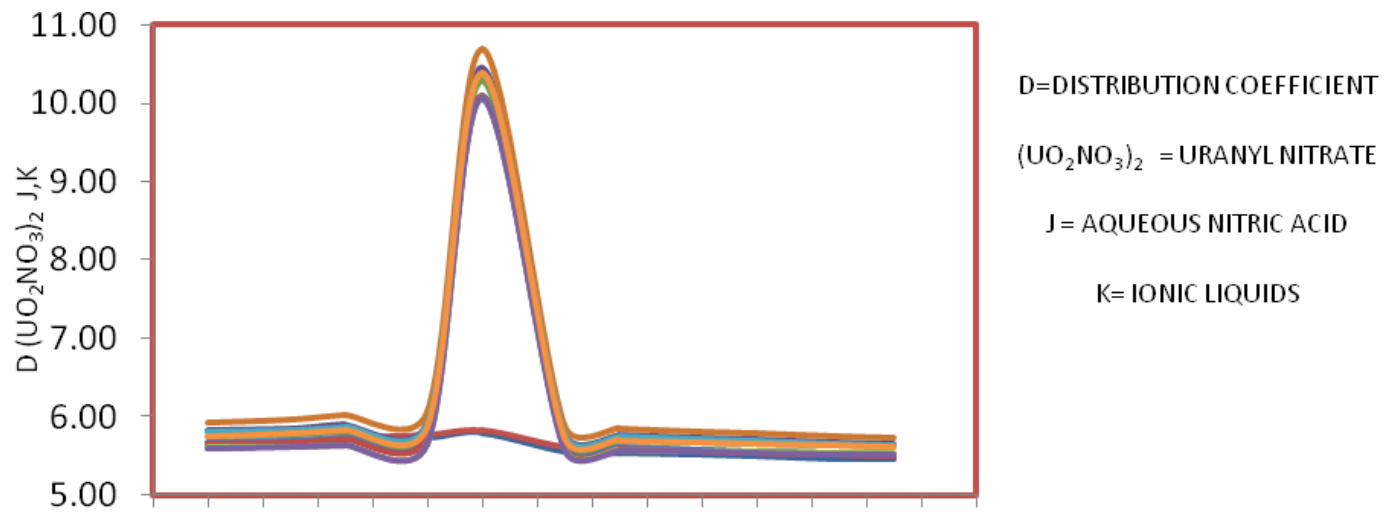

18202224262830323436384042444648

Concentration of TBP (\%)

Figure 4. Effect of TBP concentration on the $\mathrm{D}\left(\mathrm{UO}_{2} \mathrm{NO}_{3}\right)_{2} \mathrm{~J}, \mathrm{~K}$ of the selected ionic liquids.

aqueous phase decreases as a result of poor coordination of the nitrate ions with the uranyl nitrate while its solubility in the ionic liquid phase increases. Conversely, the solubility of the solute increases with the increase in nitric acid concentration as a result of its good coordination with the nitrate ions.

\section{Effect of TBP concentration on distribution coefficient}

TBP is the universally accepted extractant used in the
PUREX process for the extraction of uranium and plutonium from spent nuclear fuel. It forms complex with elements at the $+v i$ and +iv oxidation states. Uranium with the most stable oxidation state of $+v i$ forms complex with the TBP while plutonium at the +iv oxidation state also forms complex with the TBP. They are extracted together to the organic phase and then separated to obtain uranium and plutonium. As shown in Figure 4, the distribution coefficient of uranyl nitrate in ionic liquids/aqueous nitric acid biphasic system, increases with increase in TBP concentration from 20 to $30 \%$ and then decreases with increase in TBP concentration from 
30 to $45 \%$. The maximum distribution coefficient is obtained at $30 \%$ TBP concentration. This is also reported by Binnemans (2007) that optimum distribution coefficient is obtained at $30 \%$ TBP concentration from the range of percentage application of the TBP concentration from 20 to $40 \%$.

\section{Conclusion}

1) There are many potential ionic liquids that display higher distribution coefficient than n-dodecane.

2) 17 ionic liquids based on $\mathrm{BF}_{4}$ are the most recommended hydrophobic ionic liquids of the 111 screened.

3) Tributyl-phosphate TBP has effect on thedistribution coefficient. The highest distribution coefficient is obtained at $30 \%$ TBP by volume and four (4) molar concentration trioxonitric (vi) acid.

4) The effect of temperature with the distribution coefficients was also investigated. The distribution coefficients of all the ionic liquids/nitric acid biphasic system were decreasing with temperature from 25 to $100^{\circ} \mathrm{C}$.

\section{REFERENCES}

Binnemans K (2007).Lanthanides and Actinides Chemistry in ionic liquids. Chem.Rev.

Eckert F, Klamt A (2002). Fast solvent screening via quantum chemistry COSMO-RS approach. AIChE J. 48(2):369.

Felicia S, Alice M, Petre I, Adrian B, Titus C, Alexandru T B (2009). New alternatives for estimating the Octanol/water partition coefficient and water solubility for volatile organic compounds using GLC data (Kovàts retention indices). ARKIVOC (10):174-194.

Hamad EM, Sunon KZ (2008). Computational screening of ionic liquids for aromatic extraction using COSMO-RS.

Klamt A (1995). Conductor-like Screening Model for Real Solvents: A New Approach to the Quantitative Calculation of Solvation Phenomena. J. Phys. Chem. 99:2224.

Sung HH, Russel MN, Yoon MK (2010). Reprocessing of spent nuclear fuel using ionic liquid. Korean J. Chem. Eng. 27950:1360-1365

Wasserscheid P, Keim W (2000). Ionic liquids - New solutions for transition metal catalysis. Angew. Chem.-Int. Edit. 39(21):3773-3789. 